\title{
A study of the relationship between tuberculosis disease and the severity of COVID-19; a systematic review study
}

\author{
Abuzar Shirazi $^{\mathbb{D}}$, Nasrin Moradi $^{\circledR}$, Bahareh Behzadi ${ }^{3}$, Masoumeh Sadat Mousavi $^{4,5^{*}}{ }^{\mathbb{D}}$, Sadeq Hosseinpour $^{\circledR}$, \\ Ebrahim Moayedi ${ }^{1}$
}

${ }^{1}$ Department of Epidemiology and Biostatistics, School of Public Health, Shahrekord University of Medical Sciences, Shahrekord, Iran

${ }^{2} \mathrm{PhD}$ Student, School of Medical Management and information, Iran University of Medical Sciences, Tehran, Iran ${ }^{3}$ Department of Epidemiology and Biostatistics, Medical School, Rafsanjan University of Medical Sciences, Rafsanjan, Iran ${ }^{4}$ Modeling in Health Research Center, Shahrekord University of Medical Sciences, Shahrekord, Iran.

${ }^{5}$ Department of Epidemiology and Biostatistics, School of Public Health, Shahrekord University of Medical Sciences, Shahrekord, Iran

\section{Correspondence to:} Masoumeh Sadat Mousavi, Email: mousavi68.ma@yahoo. com,Mousavi.ma@skums.ac.ir

Received: 9 Feb. 2021 Accepted: 12 July 2021 ePublished: 29 Aug. 2021

\section{Keywords: COVID- 19} Tuberculosis, Systematic review, Mycobacterium tuberculosis

\begin{abstract}
Citation: Shirazi A, Moradi N, Behzadi B, Mousavi MS, Hosseinpou S, Moayedi E. A study of the relationship between tuberculosis disease and the severity of COVID-19; a systematic review study. J Prev Epidemiol. 2021;6(2):e35. doi: 10.34172/jpe.2021.35
\end{abstract}

\begin{abstract}
Introduction: Studies on COVID-19 disease in patients with tuberculosis (TB) have not yet been widely performed and clear results have not been presented. It was found that; COVID-19 infection in TB patients quickly leads to disease and causes death in a small number of them. However, the prognosis of co-infection has not been evaluated in long run, since the association between TB and covid-19 cannot be ruled out, and therefore appropriate precautions and health system preparation are needed to deal with the impending burden of co-infection. The aim of this study was to provide a clear picture of the effects of these two diseases with each other.

Methods: This study is considered as a systematic review of secondary studies. The statistical population is the studies that have been conducted in relation to the above topic and in the world. These articles were extracted from the databases of PubMed, Magiran, Science Direct, and Google Scholar. Out of 180 articles, 52 articles were related to our subject were selected, while 30 duplicates were removed from this number, hence 30 articles were selected. Finally, after a qualitative review, 12 articles were included in the study and the results of five articles were analyzed.

Results: Studies have shown that co-infection with COVID-19 and tuberculosis can increase mortality; TB is also likely to exacerbate the course of COVID-19 for the infected population and may increase the TB epidemic in the short term after the end of the COVID-19 epidemic is effective, so appropriate precautions and preparation of the health system are needed to deal with the impending burden of co-infection.

Conclusion: COVID-19 disease and its limitations may have a significant impact on the presentation and monitoring of TB control strategies nationally and globally. Concerns about delays in treating TB patients make their disease worse, and therefore broader management should be conducted.
\end{abstract}

\section{Introduction}

Tuberculosis (TB) is an infectious disease and the leading cause of death from single agent infectious diseases (1). TB and coronavirus are both epidemics that show a persistent transmission of epidemics in society and around the world, while TB is a slow pandemic that has plagued humans for millennia. COVID-19 is a new disease and is spreading rapidly around the world. TB seems to be an important disease for the deterioration of the clinical status of COVID-19 cases, since the isolation of cases of TB can be an important criterion for minimizing the occurrence of severe cases of COVID-19 and hospitalization due to the disease in this population even in conditions of isolation (2). In late 2019,

\begin{abstract}
Key point
COVID-19 disease and its limitations may have a significant impact on the presentation and monitoring of TB control strategies nationally and globally. Concerns about delays in treating TB patients make their disease worse, and therefore broader management should be conducted.
\end{abstract}

an acute respiratory illness caused by a new coronavirus called Severe acute respiratory syndrome coronavirus 2 (SARS-CoV-2) broke out in Wuhan, China. The disease spread rapidly in cities in China and other countries. The disease continued to worsen as the World Health Organization (WHO) declared a state of emergency in connection with the disease on January 30, 2020, and 
on February 12, 2020, the new coronavirus disease was named coronavirus 2019 or COVID-19. The power of transmission of this disease is very high and due to the dangers and lethality of this virus, the best way to fight this disease is to control the disease and prevent the eradication of both cases. In the other words, to cut the transmission chain of the disease and to achieve this. Hence, the goal is to have a detailed plan to control and prevent the disease (3). Manifestations of the disease range from mild cough, fever and sore throat to pneumonia, acute respiratory distress syndrome (ARDS), and death. Patients with COVID-19 cases are more likely to develop a severe illness (4). Clinical manifestations of COVID-19 range from asymptomatic to severe symptoms requiring admission to an intensive care unit. The presentations of COVID-19 can be mild to severe with various respiratory complications, gastrointestinal, hepatic, and neurological disorders (5). TB is a persistent chronic bacterial disease that is highly prevalent in developing countries. The WHO estimates that 1.7 billion people worldwide are infected with Mycobacterium tuberculosis and are therefore at risk for the disease (4). Approximately 1.5 million deaths from TB occur worldwide each year, making it the tenth most common disease in the world and is expected to maintain its ranking by 2020. On the other hand, information about death and its causes is necessary to assess the health status of the region and to analyze health programs and interventions (6). Co-infection of TB with coronavirus such as SARS and the Middle East respiratory syndrome (MERS) virus. Has been a great threat (4). The clinical features and treatment of pulmonary TB patients with COVID-19 are unclear and have not been studied (7). Some viral infections, such as measles, have been shown to exacerbate pulmonary TB due to a weakened immune system, but despite the vulnerability of this type of population, so far most COVID-19 studies have focused on patients without respiratory disease (7). There is limited information on the risk of severe disease or complications in patients with TB and COVID-19 (8). Therefore, considering that, COVID-19 disease is one of the emerging diseases and sufficient information about the behavior of the disease and long-term complications and its effect on other diseases is still unknown. This study aims to reach a general conclusion by examining the results of several studies on the course of COVID-19 disease, in TB patients.

\section{Methods}

This is a systematic secondary study of the review type. Reviewing past research is done in different ways, one of the most well-known of which is systematic review, which is a kind of secondary study and analysis of studies. In other words, systematic review is a structured search that is performed according to predetermined rules and regulations. The statistical population of this systematic study includes all research articles on COVID-19 in patients with $\mathrm{TB}$, which from the beginning of the pandemic COVID-19 published so far.

\section{Search strategy}

PubMed, Magiran, Science Direct, SID, Google Scholar databases were selected for searching related articles and advanced search option was used with keywords and, or operators in these databases. Finally, out of 180 searches (125 articles in PubMed, 15 articles in science direct, 40 articles in google scholar.) 52 related articles were selected, duplicates selected in the databases were removed, and from this number, 30 articles were selected, and according to the study of the abstracts of the mentioned articles, some of them are related to vaccination TB and treatment of TB was eliminated due to its lack of relevance to the subject, and finally, after a qualitative review, 12 articles were included in the study and the results of 5 articles were analyzed (Figure 1).

\section{Results}

In the study conducted by Tadolin et al on a group of
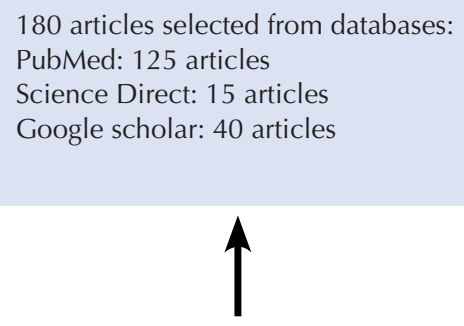

Finally, after a qualitative review, 12 articles (5 articles from PubMed, 6 articles from Google Scholar, 1 article from Science Direct) which was about COVID-19 and TB, were included in the study and the results of 5 articles were analyzed.
52 articles (13 articles from PubMed, 70 articles from science direct, 22 articles from google scholar) with title appropriate to the subject of the article were selected and other items were deleted

Re-examinations were performed, duplicates were removed, and 30 articles were selected

Figure 1. Summary of search strategy and paper exclusion. 
49 patients, $53 \%$ of the participants in the study had a history of TB, 28.5\% initially had COVID-19 and 18.3\% had both TB and COVID-19 at the same time. In $8.38 \%$ of patients, COVID-19 appeared during TB treatment, indicating the potential risk of transmission of the disease to patients, which led to the spread of the hospital and the prevalence of COVID-19 in health staff, and showed that infection and severity of COVID-19 were present in patients with active and latent TB infections are more likely (4). Additionally, in a study conducted by Motta et al in Italy in two groups of patients infected with TB and COVID-19, about 69 patients in both groups (11.4\%) were exposed to a common infection. COVID-19 infection in TB patients rapidly leads to the death of a small number of them. However, the prognosis of long-term concomitant infection has not been evaluated in this study. Although these studies showed early manifestations, the association between TB and COVID-19 cannot be ruled out, and therefore appropriate precautions and preparation of the health system to deal with the impending burden of coinfection are needed (4). A study by Crisan-Dabija et al, found that people with chronic respiratory infections, including TB, experienced their side effects for the first time, especially in a healthcare setting. We are looking at the possible consequences of the inevitable collision of two epidemic diseases. The destructive synergy of viral and bacterial infections increases mortality. In fact, patients with pulmonary $\mathrm{TB}$ due to chronic lung disease and immune suppression due to a viral disease like COVId-19 or influenza experience an increased mortality (11). He et al, in their study on three patients with TB and COVID-19 at the same time, stated that all three patients were male and aged 26 to 74 years. All patients were followed up prospectively from admission to discharge from the hospital. All patients had close contact and others have been infected with confirmed infections of the COVID-19. Patients 1 and 2 had a history of pulmonary TB and were and receiving anti-TB treatment, while patient 3 had not been treated for TB. Patient one progressed to type 1 respiratory failure and ARDS within 10 days of onset and was treated with non-invasive ventilation support. Both patients one and three were in severe cases and were treated with methylprednisolone. Additionally, patient 2 had skin rashes with itching all over his body, which is a side effect of antiviral therapy. His symptoms improved after stopping antiviral treatment. All patients recovered and were discharged from the hospital. However, on day 9 after discharge, a patient had two positive recurrences of RNA SARS-CoV-2 and therefore returned to the hospital to be isolated and monitored (7). In a study conducted in China on March 11, 2020, Hong Yang et al showed, SARS-CoV-2 and immunosuppressive drugs temporarily inhibit immune function, resulting in active TB caused by recurrence or new infection with Mycobacterium in addition to the likely exacerbating the course of COVID-19 (8).

\section{Discussion}

A review of studies related to COVID-19 in TB patients showed that in patients with $\mathrm{TB}$ the induction of type 1 interferons could be harmful due to the weakening of the immune system due to the inability of the host to limit bacterial replication (11). In contrast, few studies have shown that no association between infection and progression and severity of pulmonary TB was existed (4). The administration of corticosteroids in the treatment of SARS has led to the suppression of the immune system. Transient suppression of the immune system has shown two spectrums of the disease and it is clear that people have recovered from SARS, infected with COVID-19 and vice versa.

\section{Key findings of the study}

1- Prevalence of seasonal influenza and other coronavirus epidemics may have a negative impact on TB patients.

2- Prevention of transmission is critical to control epidemics.

3- Lack of access to appropriate treatment conditions SARS-CoV-2 in TB cases makes patients worse. It should be kept in mind that the presence of rheumatic diseases, autoimmune diseases, poor hygiene, and overcrowding are risk factors for the development of other diseases or both, which is another serious problem caused by the COVID-19 epidemic.

\section{Continuation of treatment of TB patients}

There is evidence that BCG vaccination in adults can increase the statistical capacity to produce cytokines such as Il-1 $\beta$ and IL-6, leading to nonspecific protection against unrelated pathogens such as Staphylococcus aureus or Candida albicans. Given these facts, the BCG vaccine is considered as a potential candidate against respiratory viruses (11). In patients infected with Mycobacterium tuberculosis, whether treated or untreated, various types of usury and extra-pulmonary and structural complications lead to obstruction, restriction, or mixing of pulmonary dysfunction. Studies in patients with pulmonary TB have shown that $33.3-94 \%$ of these patients develop pulmonary dysfunction. Risk factors for patients with reduced lung function are having a history of pulmonary TB, positive culture of this disease and patients over 50 years, low-level of education and experience of recurrence TB. The study shows that previous lung diseases such as Mycobacterium tuberculosis treated or untreated and old age are independent risk factors for a worse prognosis in people infected with COVID-19. In this study, elderly patients with TB easily progressed to the severe form of COVID-19 and had a long recovery process (7). Challenges and strategies for operating management during COVID-19 pandemic disease include (a) Decrease in diagnosis of new cases of active TB. (b) Challenges of out-of-patient regulation. (c) Challenges of TB in the society. (d) Contact tracking programs. (e) Monitoring and supply of anti-TB drugs. 
Table 1. Review of texts

\begin{tabular}{|c|c|c|c|c|c|}
\hline Author Name & Location & Population & $\begin{array}{c}\text { Number of } \\
\text { people surveyed }\end{array}$ & Type of study & Result \\
\hline Tadolini et al (9) & Italy & $\begin{array}{l}\text { People with } \\
\text { TB, people with } \\
\text { COVID-19 and } \\
\text { people with both } \\
\text { diseases }\end{array}$ & 49 & Cohort & $\begin{array}{l}\text { In } 38.8 \% \text { of patients COVID-19 appeared during TB treatment, } \\
\text { indicating a potential risk of transmission of the disease to } \\
\text { caregivers, which led to hospital outbreaks and the prevalence } \\
\text { of COVID-19 in health care workers. Active and latent are more } \\
\text { likely. }\end{array}$ \\
\hline Motta et al (10) & Italy & $\begin{array}{l}\text { Two groups of } \\
\text { patients with TB } \\
\text { and COVID-19 }\end{array}$ & 69 & Cohort & $\begin{array}{l}\text { COVID-19 infection has been shown to rapidly kill a small number } \\
\text { of TB patients. However, the prognosis of long-term concomitant } \\
\text { infection has not been evaluated in this study. Although these } \\
\text { studies showed early manifestations, the association between TB } \\
\text { and COVID-19 cannot be ruled out, and therefore appropriate } \\
\text { precautions and preparation of the health system are needed to } \\
\text { deal with the impending burden of co-infection. }\end{array}$ \\
\hline $\begin{array}{l}\text { Crisan-Dabija et } \\
\text { al(11) }\end{array}$ & Romania & - & - & Systematic & $\begin{array}{l}\text { The destructive synergy of viral and bacterial infections increases } \\
\text { mortality and patients with pulmonary TB due to chronic lung } \\
\text { disease and suppression of the immune system increase the severity } \\
\text { of viral diseases such as influenza and mortality in them. }\end{array}$ \\
\hline He et al (7) & China & $\begin{array}{l}\text { Patients with both } \\
\text { TB and COVID-19 } \\
\text { at the same time }\end{array}$ & 3 & Case report & $\begin{array}{l}\text { Among the three patients with the combination of TB and } \\
\text { COVID-19, two older patients developed severe cases of } \\
\text { COVID-19 and the other had symptoms of ARDS (respiratory } \\
\text { distress syndrome). Each patient had a long recovery and it was } \\
\text { difficult to solve their low-oxygen index problem }\end{array}$ \\
\hline Yang et al (8) & China & $\begin{array}{l}\text { People with } \\
\text { TB, people with } \\
\text { COVID-19 and } \\
\text { people with both } \\
\text { diseases }\end{array}$ & - & Review & $\begin{array}{l}\text { This study shown both effects of SARS-CoV- } 2 \text { and } \\
\text { immunosuppressive drugs on temporarily inhibition of immune } \\
\text { function and outcome of active TB due to recurrence or new } \\
\text { infection with Mycobacterium. In addition, there is an exacerbating } \\
\text { course of COVID-19 disease by Mycobacterium in the infected } \\
\text { population. }\end{array}$ \\
\hline
\end{tabular}

(f) To test COVID-19 and TB. (g) Disease COVID-19 and TB. $(h)$ Closing effects during pandemic. (i) Personal protection equipment COVID-19. 10- Prevention of drugresistant aerosol. ( $j$ ) Personal safety of health care workers. (k) Epidemic impact COVID-19 on TB immunization program. (l) Economic consequences of pandemic COVID-19 (12). TB continues to be a major cause of infectious respiratory disease that threatens public health. There is limited information on the risk of severe disease or outcomes in patients with TB and COVID-19 (8). In most patients, SARS-CoV-2 infection was nosocomial and required precise infection prevention and control measures to prevent cross-infection. It was found that COVID-19 infection in TB patients quickly leads to the death of a small number of them (4). According to the above points, although not enough studies have been conducted on this subject and the unknown dimensions of the disease and its relationships and effects It is not yet known, but given the incomplete information currently available as well as comparisons with the behavior of other viruses in the coronavirus family, the statement that the two diseases are transmissible can be reinforced and confirmed.

\section{Conclusion}

Viral respiratory infections and $\mathrm{TB}$ prevent host immune responses, suggesting that their lethal synergy may contribute to more intense clinical development.
COVID-19 disease and its limitations may have a significant impact on the presentation and monitoring of TB control strategies nationally and globally. Concerns about delays in treating TB patients make their disease worse, and therefore broader management should be conducted. In addition, TB patients may develop multidrug resistance and extremely acute infections with the coronavirus, since the effect is that COVID-19 and immunosuppressive drugs may weaken immune function and exacerbate the disease process for patients. We should be sensitive to the short-term increase of the TB epidemic after the end of the COVID-19 epidemic. This requires not only TB prevention and control training, but also adequate measures to strengthen TB prevention, control and management. According to the current studies the destructive effects of TB and COVID-19 diseases on each other, it is recommended to strengthen the education of TB patients in the COVID-19 pandemic.

\section{Authors' contribution}

AS, MSM, SH and EM contributed to the search and analysis of the data. MSM is the corresponding author which conducted the final edit. All authors read and signed the final paper.

Conflicts of interest

The authors declare that they have no competing interests.

Ethical issues

Ethical issues (including plagiarism, data fabrication, double 
publication) have been completely observed by the authors.

Funding/Support

None.

\section{References}

1. MofarahZat A, Fazaeli AA, Hamidi Y. Study of socioeconomic factors affecting tuberculosis and its cost in Hamadan province. Hospital. 2020;19:9-16.

2. Joob B, Wiwanitkit V. Letter to the Editor: Coronavirus Disease 2019 (COVID-19), Infectivity, and the Incubation Period. J Prev Med Public Health. 2020;53:70. doi: 10.3961/jpmph.20.065.

3. Golahdooz M, Tabibzadeh, Taherizadeh M, Laali A, Khoonsari M, Ajdarkosh H, et al . A review on Coronavirus 2019 Disease (COVID-19, SARS-CoV-2): Control and Prevention. Razi J Med Sci. 2020;27:98-107

4. Bandyopadhyay A, Palepu S, Bandyopadhyay K, Handu S. COVID-19 and tuberculosis co-infection: a neglected paradigm. Monaldi Arch Chest Dis. 2020;90(3). doi: 10.4081/ monaldi.2020.1437.

5. Seif F, Aazami H, Khoshmirsafa M, Kamali M, Mohsenzadegan M, Pornour M, Mansouri D. JAK Inhibition as a New Treatment Strategy for Patients with COVID-19. Int Arch Allergy Immunol. 2020;181:467-475. doi: 10.1159/000508247.

6. Jafarvand A, Ataie A. Epidemiology and death trend of tuberculosis in Iran. Scientific-Research Journal of Sabzevar Univ Med Sci. 2019;25:773-80

7. He G, Wu J, Shi J, Dai J, Gamber M, Jiang X, et al. COVID-19 in tuberculosis patients: A report of three cases. J Med Virol. 2020;92:1802-6. doi: 10.1002/jmv.25943.

8. Yang H, Lu S. COVID-19 and Tuberculosis. J TransI Int Med. 2020;8:59-65. doi: 10.2478/jtim-2020-0010.

9. Tadolini M, Codecasa LR, García-García JM, Blanc FX, Borisov $\mathrm{S}$, Alffenaar JW, et al. Active tuberculosis, sequelae and COVID-19 co-infection: first cohort of 49 cases. Eur Respir J. 2020;56:2001398. doi: 10.1183/13993003.01398-2020.

10. Motta I, Centis R, D'Ambrosio L, García-García JM, Goletti D, Gualano G, Lipani F, Palmieri F, et al. Tuberculosis, COVID-19 and migrants: Preliminary analysis of deaths occurring in 69 patients from two cohorts. Pulmonology. 2020;26:233-240. doi: 10.1016/j.pulmoe.2020.05.002.

11. Crisan-Dabija R, Grigorescu C, Pavel CA, Artene B, Popa IV, Cernomaz A, Burlacu A. Tuberculosis and COVID-19: Lessons from the Past Viral Outbreaks and Possible Future Outcomes. Can Respir J. 2020;2020:1401053. doi: 10.1155/2020/1401053.

12. Jain $V K$, lyengar KP, Samy DA, Vaishya R. Tuberculosis in the era of COVID-19 in India. Diabetes Metab Syndr. 2020;14:143943. 